Supporting Information for

\title{
Enzymatic Mechanism of Fe-only Hydrogenase: Density Functional Study on H-H Formation at the Diiron cluster with Concerted Proton and Electron Transfers ${ }^{\dagger}$
}

Taijin Zhou*, Yirong $\mathrm{Mo}^{\dagger}$, Aimin Liu ${ }^{\ddagger}$, Zhaohui Zhou and K. R. Tsai

Department of Chemistry, and the State Key Laboratory for Physical Chemistry of the

Solid Surface, Xiamen University, Xiamen 361005, P. R. China

* To whom Correspondence should be addressed. Fax: +86-592-2183795. E-mail:

tjzhou@xmu.edu.cn

$\dagger$ Permanent address: Department of Chemistry, Western Michigan University, Kalamazoo, MI 49008, USA.

$\$$ Permanent address: Department of Biochemistry, University of Mississippi Medical Center, Jackson, MS 39216, USA. 
Table S1. The optimized geometry of model $1[-2,0]^{*}$ in Cartesian coordinates

\begin{tabular}{rrrr}
\hline Fe 0 & 0.796997 & -1.211502 & -0.194992 \\
Fe O & -1.696991 & -0.735504 & 0.212006 \\
S O & -0.476990 & 0.150482 & -1.498978 \\
S O & -0.012985 & -0.024506 & 1.568008 \\
C O & -2.422989 & -1.573502 & 1.729004 \\
N $~$ & -2.908997 & -2.068497 & 2.681000 \\
C O & 1.225998 & -2.225494 & -1.717987 \\
N 0 & 1.636993 & -2.771500 & -2.680984 \\
C 0 & 1.211000 & -2.574493 & 0.803009 \\
O O & 1.545990 & -3.507492 & 1.430008 \\
C O & -3.139984 & 0.191483 & -0.048004 \\
O O & -4.156998 & 0.730484 & -0.301987 \\
C O & -1.900986 & -2.217500 & -0.687988 \\
O O & -2.150986 & -3.211502 & -1.249985 \\
C O & 0.364990 & 1.742493 & 1.276000 \\
C O & -0.494995 & 2.401489 & 0.235000 \\
C O & -0.197998 & 1.929489 & -1.160980 \\
S O & 2.711000 & -0.154495 & -0.291000 \\
C O & 3.105988 & 0.219482 & -2.034988 \\
H O & 1.436996 & 1.814484 & 0.990997 \\
H O & 0.235000 & 2.246490 & 2.255005 \\
H O & -1.560989 & 2.195480 & 0.468002 \\
H O & -0.344986 & 3.507492 & 0.276000 \\
H O & 0.866989 & 2.144485 & -1.401978 \\
H O & -0.823990 & 2.483490 & -1.889984 \\
H O & 3.692993 & -1.143494 & -0.244003 \\
H O & 4.156998 & 0.548492 & -2.131989 \\
H O & 2.421997 & 1.029495 & -2.339981 \\
H O & 2.883987 & -0.683502 & -2.631989 \\
& & & \\
\hline
\end{tabular}

${ }^{*} \mathbf{x}[\mathrm{x} 1, \mathrm{x} 2]$ express a model $\mathbf{x}$, where $\mathrm{x} 1$ denotes value of charge, and $\mathrm{x} 2$ is value of spin 
Table S2. The optimized geometry of model $2[-1,0]$ in Cartesian coordinates

\begin{tabular}{lrrr}
\hline Fe 0 & -2.027786 & -1.315491 & -0.495026 \\
Fe O & 0.456085 & -1.322495 & 0.127335 \\
S O & -0.418976 & -0.237518 & -1.680832 \\
S O & -1.143326 & -0.290512 & 1.348587 \\
C O & -2.932129 & -2.452500 & 0.698318 \\
N O & -3.511734 & -3.149490 & 1.442398 \\
C O & 1.534851 & -2.306503 & -1.046371 \\
N O & 2.296951 & -2.874496 & -1.738663 \\
C O & 0.890396 & -2.407500 & 1.419968 \\
O O & 1.227554 & -3.136505 & 2.258728 \\
C O & -2.381729 & -2.399490 & -1.829086 \\
O O & -2.664368 & -3.103500 & -2.702042 \\
C O & -3.447433 & -0.330521 & -0.738388 \\
O O & -4.433914 & 0.256485 & -0.919968 \\
S O & 2.076218 & 0.135483 & 0.455536 \\
C O & 3.642578 & -0.765503 & 0.557297 \\
C O & -0.997757 & 1.512482 & 1.102859 \\
C O & -1.345978 & 2.038483 & -0.260635 \\
C O & -0.437790 & 1.554489 & -1.353394 \\
H O & -0.731537 & -2.376495 & -0.219620 \\
H O & 2.043655 & 0.460480 & 1.796967 \\
H O & 4.477631 & -0.060516 & 0.695175 \\
H O & 3.723694 & -1.309494 & -0.399811 \\
H O & 3.600494 & -1.505493 & 1.371200 \\
H O & 0.042862 & 1.797485 & 1.362198 \\
H O & -1.658905 & 1.967484 & 1.862671 \\
H O & -1.295029 & 3.149490 & -0.229324 \\
H O & -2.392395 & 1.777481 & -0.500717 \\
H O & 0.611664 & 1.835480 & -1.129044 \\
H O & -0.709885 & 2.030487 & -2.311935 \\
\hline
\end{tabular}


Table S3. The optimized geometry of model 3[0,0] in Cartesian coordinates

\begin{tabular}{lrrr}
\hline Fe O & -2.119492 & -0.937988 & -0.341003 \\
Fe O & 0.305496 & -1.272980 & 0.514984 \\
S O & -0.322495 & 0.080002 & -1.101990 \\
S O & -1.277496 & -0.082000 & 1.623993 \\
C O & -3.142487 & -2.055984 & 0.747986 \\
N O & -3.752487 & -2.761978 & 1.455994 \\
C O & 1.344498 & -2.207977 & -0.718002 \\
N O & 2.035492 & -2.686981 & -1.537994 \\
C O & 0.397491 & -2.577988 & 1.683990 \\
O O & 0.493500 & -3.451981 & 2.427994 \\
C O & -2.503494 & -1.950989 & -1.737991 \\
O O & -2.781494 & -2.618988 & -2.630997 \\
C O & -3.473495 & 0.168000 & -0.550995 \\
O O & -4.392487 & 0.854004 & -0.669998 \\
S O & 2.084488 & -0.184006 & 1.268982 \\
C O & 3.494492 & -1.309982 & 1.282990 \\
C O & -0.883484 & 1.707000 & 1.487991 \\
C O & -1.085495 & 2.362000 & 0.147003 \\
C O & -0.162506 & 1.877000 & -0.938995 \\
H O & -0.965485 & -2.134979 & -0.031998 \\
H O & 0.252487 & -0.126007 & -2.334000 \\
H O & 1.923492 & -0.123000 & 2.630981 \\
H O & 4.392502 & -0.778992 & 1.632996 \\
H O & 3.615494 & -1.643982 & 0.240005 \\
H O & 3.275497 & -2.185989 & 1.909988 \\
H O & 0.159500 & 1.839005 & 1.834991 \\
H O & -1.536484 & 2.190002 & 2.233994 \\
H O & -0.924484 & 3.451996 & 0.258987 \\
H O & -2.135498 & 2.233994 & -0.173004 \\
H O & 0.898500 & 2.087997 & -0.707993 \\
H O & -0.420486 & 2.324005 & -1.914000 \\
\hline
\end{tabular}


Table S4. The optimized geometry of model $4[-2,0]$

\begin{tabular}{lrrr}
\hline Fe 0 & -2.077988 & -0.795273 & -0.419952 \\
Fe O & 0.360000 & -1.263062 & 0.177795 \\
S O & -0.468979 & 0.446075 & -1.405228 \\
S O & -1.141983 & -0.397705 & 1.605377 \\
C O & -3.030975 & -2.136139 & 0.460403 \\
N $~$ & -3.594986 & -2.990311 & 1.030289 \\
C O & 1.505005 & -1.931686 & -1.169600 \\
N $~$ & 2.309006 & -2.353897 & -1.915039 \\
C O & 0.539000 & -2.773819 & 1.041946 \\
O O & 0.699997 & -3.789139 & 1.559586 \\
C O & -2.631989 & -1.480469 & -1.952484 \\
O O & -3.039978 & -1.942337 & -2.922913 \\
C O & -3.415985 & 0.338226 & -0.263763 \\
O O & -4.325989 & 1.036118 & -0.149673 \\
S O & 2.074005 & -0.238510 & 1.157913 \\
C O & 3.507996 & -1.332382 & 1.155548 \\
C O & -0.765976 & 1.374634 & 1.832169 \\
C O & -0.934982 & 2.282059 & 0.645691 \\
C O & -0.039993 & 1.985229 & -0.523987 \\
H O & -0.898987 & -2.009567 & -0.518906 \\
H O & 1.798004 & -0.350571 & 2.497940 \\
H O & 4.326004 & -0.861679 & 1.721786 \\
H O & 3.792007 & -1.464005 & 0.098831 \\
H O & 3.240997 & -2.313385 & 1.575760 \\
H O & 0.268005 & 1.443512 & 2.223282 \\
H O & -1.434982 & 1.699219 & 2.648987 \\
H O & -0.720978 & 3.319016 & 0.977173 \\
H O & -1.990982 & 2.282349 & 0.320313 \\
H O & 1.025009 & 1.935486 & -0.228104 \\
H O & -0.138000 & 2.771469 & -1.292587 \\
H O & 0.776000 & -0.615555 & -1.345703 \\
& & & \\
\hline
\end{tabular}


Table S5. The optimized geometry of model 5[0,0]

\begin{tabular}{lrrr}
\hline Fe O & -2.105988 & -0.836990 & -0.153503 \\
Fe O & 0.378006 & -1.407990 & 0.446503 \\
S O & -0.316986 & 0.069000 & -1.186493 \\
S O & -1.126984 & -0.278992 & 1.779480 \\
C O & -3.110977 & -2.061981 & 0.838486 \\
N O & -3.721985 & -2.846985 & 1.460480 \\
C O & 1.721008 & -2.045990 & -0.695496 \\
N O & 2.630005 & -2.389984 & -1.350494 \\
C O & 0.699005 & -2.587982 & 1.726486 \\
O O & 0.996002 & -3.318985 & 2.564484 \\
C O & -2.700989 & -1.612976 & -1.630493 \\
O O & -3.160980 & -2.098984 & -2.564500 \\
C O & -3.385986 & 0.356995 & -0.169495 \\
O O & -4.281982 & 1.083008 & -0.174500 \\
S O & 2.052002 & -0.093994 & 1.223480 \\
C O & 3.533997 & -1.077988 & 1.510483 \\
C O & -0.724976 & 1.503006 & 1.790482 \\
C O & -0.874985 & 2.253998 & 0.498505 \\
C O & -0.007004 & 1.770004 & -0.625504 \\
H O & -0.961975 & -2.217987 & -0.110504 \\
H O & -0.016998 & -2.593979 & -0.433502 \\
H O & 1.750000 & 0.067000 & 2.551483 \\
H O & 4.281998 & -0.435989 & 1.999481 \\
H O & 3.889008 & -1.404984 & 0.519485 \\
H O & 3.304000 & -1.959976 & 2.126480 \\
H O & 0.301010 & 1.619003 & 2.192490 \\
H O & -1.406982 & 1.923004 & 2.551483 \\
H O & -0.630981 & 3.319000 & 0.693481 \\
H O & -1.931976 & 2.234009 & 0.179504 \\
H O & 1.067000 & 1.835007 & -0.364502 \\
H O & -0.163986 & 2.395004 & -1.522507 \\
\hline
\end{tabular}


Table S6. The optimized geometry of model $6[0,0]$

\begin{tabular}{lrrr}
\hline Fe O & 0.158997 & -1.075989 & 0.255997 \\
Fe O & -2.248000 & -0.560989 & -0.328995 \\
S O & -0.506989 & 0.684982 & -1.071000 \\
S O & -1.367996 & -0.350998 & 1.774994 \\
C O & -3.472000 & -1.778992 & 0.405000 \\
N O & -4.233994 & -2.528992 & 0.884979 \\
C O & 1.341995 & -1.559998 & -1.110992 \\
N O & 2.152985 & -1.813995 & -1.919998 \\
C O & 0.576996 & -2.494995 & 1.211990 \\
O O & 0.909988 & -3.410995 & 1.823990 \\
C O & -2.860992 & -0.836990 & -1.966003 \\
O O & -3.265000 & -1.048996 & -3.018997 \\
C O & -1.133987 & -2.156998 & -0.638992 \\
O O & -1.265000 & -3.207993 & -1.125000 \\
C O & -1.023987 & 1.397995 & 2.159988 \\
C O & -1.233994 & 2.404984 & 1.063980 \\
C O & -0.348007 & 2.233994 & -0.136002 \\
S O & 1.905991 & 0.054993 & 1.153992 \\
C O & 3.406998 & -0.933000 & 1.010986 \\
H O & -3.031998 & 0.897995 & -0.160004 \\
H O & -3.591995 & 0.288986 & 0.069000 \\
H O & 0.017990 & 1.456985 & 2.528992 \\
H O & -1.677994 & 1.631989 & 3.018982 \\
H O & -1.043000 & 3.410995 & 1.487991 \\
H O & -2.295990 & 2.411987 & 0.752991 \\
H O & 0.717987 & 2.303986 & 0.155000 \\
H O & -0.539000 & 3.037994 & -0.869995 \\
H O & 1.756989 & -0.112015 & 2.506989 \\
H O & 4.233994 & -0.388000 & 1.489990 \\
H O & 3.590988 & -1.052002 & -0.069000 \\
H O & 3.268997 & -1.927002 & 1.462982 \\
\hline
\end{tabular}


Table S7. The optimized geometry of model 7[-1,1/2]

\begin{tabular}{lccc}
\hline Fe O & -1.980927 & -0.302490 & -0.480621 \\
S O & -0.394241 & 0.752502 & -1.668365 \\
S O & -1.102509 & 0.663498 & 1.341675 \\
C O & -2.664932 & -1.562485 & 0.719742 \\
N O & -3.087906 & -2.356491 & 1.477173 \\
C O & -2.090347 & -1.541489 & -1.719696 \\
O O & -2.217102 & -2.368484 & -2.520096 \\
C O & -3.463058 & 0.556503 & -0.804077 \\
O O & -4.462784 & 1.104492 & -1.044144 \\
C O & -0.953140 & 2.472488 & 1.129974 \\
C O & -1.299194 & 3.011490 & -0.229141 \\
C O & -0.388535 & 2.545502 & -1.329102 \\
Fe 0 & 0.486832 & -0.340485 & 0.091034 \\
C O & 1.622635 & -1.284485 & -1.054291 \\
N O & 2.450653 & -1.809494 & -1.707550 \\
C O & 0.682449 & -1.629486 & 1.269272 \\
O O & 0.868195 & -2.490494 & 2.025406 \\
S O & 2.080688 & 1.049500 & 0.635895 \\
C O & 3.629135 & 0.140518 & 0.845000 \\
H O & 0.082275 & 2.760498 & 1.407669 \\
H O & -1.627640 & 2.908493 & 1.890000 \\
H O & -1.259247 & 4.121490 & -0.187500 \\
H O & -2.341568 & 2.735489 & -0.475586 \\
H O & 0.664642 & 2.817490 & -1.113037 \\
H O & -0.668427 & 3.025497 & -2.284302 \\
H O & 1.901200 & 1.318497 & 1.976837 \\
H O & 4.447418 & 0.829498 & 1.113449 \\
H O & 3.817154 & -0.336487 & -0.132492 \\
H O & 3.502060 & -0.652496 & 1.598343 \\
H O & -0.566422 & -4.121490 & -0.697632 \\
H O & -0.421448 & -3.382492 & -0.538467 \\
\hline
\end{tabular}


Table S8. The optimized geometry of model $\mathbf{8}[-1,1 / 2]$

\begin{tabular}{lrrr}
\hline Fe 0 & 0.291489 & -1.082489 & 0.226500 \\
Fe O & -2.203491 & -0.559494 & -0.363495 \\
S O & -0.434494 & 0.687500 & -1.100494 \\
S O & -1.262482 & -0.376480 & 1.759491 \\
C O & -3.501495 & -1.716492 & 0.356506 \\
N O & -4.355484 & -2.390488 & 0.795486 \\
C O & 1.494492 & -1.556488 & -1.132492 \\
N 0 & 2.344498 & -1.812485 & -1.901489 \\
C O & 0.724503 & -2.477493 & 1.174484 \\
O O & 1.062500 & -3.401489 & 1.792496 \\
C O & -2.826492 & -0.818481 & -1.972504 \\
O O & -3.251495 & -1.033493 & -3.029495 \\
C O & -1.061493 & -2.121490 & -0.636490 \\
O O & -1.192490 & -3.191483 & -1.110489 \\
C O & -0.913483 & 1.373505 & 2.150482 \\
C O & -1.160492 & 2.390503 & 1.068481 \\
C O & -0.287491 & 2.234497 & -0.146500 \\
S O & 2.022491 & 0.141495 & 1.096481 \\
C O & 3.500504 & -0.897491 & 1.029495 \\
H O & -3.493484 & 0.362503 & 0.142502 \\
H O & 0.140503 & 1.445496 & 2.488495 \\
H O & -1.543488 & 1.598495 & 3.029495 \\
H O & -0.982483 & 3.401505 & 1.496490 \\
H O & -2.225494 & 2.369492 & 0.771484 \\
H O & 0.779495 & 2.321503 & 0.142502 \\
H O & -0.496490 & 3.044495 & -0.868500 \\
H O & 1.877502 & 0.051498 & 2.465485 \\
H O & 4.355500 & -0.356491 & 1.466492 \\
H O & 3.659500 & -1.105484 & -0.043503 \\
H O & 3.321503 & -1.853485 & 1.546494 \\
H O & -2.986496 & 0.942505 & -0.216492 \\
\hline & & &
\end{tabular}


Table S9. The optimized geometry of model 9[-1,0]

\begin{tabular}{lrrr} 
Fe O & 0.315994 & -1.080978 & 0.188004 \\
Fe O & -2.063995 & -0.597992 & -0.384995 \\
S O & -0.369995 & 0.688004 & -1.134003 \\
S O & -1.229996 & -0.355988 & 1.711990 \\
C O & -3.442000 & -1.606979 & 0.369995 \\
N O & -4.328995 & -2.208984 & 0.851990 \\
C O & 1.495987 & -1.589981 & -1.183000 \\
N O & 2.329987 & -1.856979 & -1.966995 \\
C O & 0.718994 & -2.490982 & 1.135986 \\
O O & 1.066986 & -3.402985 & 1.765991 \\
C O & -2.839996 & -0.610992 & -1.938004 \\
O O & -3.405000 & -0.577988 & -2.949005 \\
C O & -1.113998 & -2.193985 & -0.716995 \\
O O & -1.151993 & -3.276993 & -1.160004 \\
C O & -0.950989 & 1.408005 & 2.085983 \\
C O & -1.237991 & 2.365005 & 0.965988 \\
C O & -0.285995 & 2.240997 & -0.188004 \\
S O & 1.984985 & 0.106995 & 1.081985 \\
C O & 3.493988 & -0.886978 & 1.012985 \\
H O & -3.095000 & 0.516998 & -0.218994 \\
H O & 0.095993 & 1.530000 & 2.434982 \\
H O & -1.606995 & 1.619995 & 2.948990 \\
H O & -1.186996 & 3.403000 & 1.358978 \\
H O & -2.272003 & 2.188004 & 0.606979 \\
H O & 0.760986 & 2.350998 & 0.160995 \\
H O & -0.472992 & 3.039993 & -0.927994 \\
H O & 1.816986 & -0.024994 & 2.443985 \\
H O & 4.328995 & -0.323990 & 1.457977 \\
H O & 3.665985 & -1.081985 & -0.059998 \\
H O & 3.338989 & -1.849991 & 1.523987 \\
\hline
\end{tabular}


Table S10. The optimized geometry of model $\mathbf{1 0}[0,0]$

\begin{tabular}{lrrr}
\hline Fe O & -1.802994 & -0.794479 & 0.531006 \\
Fe O & 0.532990 & -1.136490 & -0.371979 \\
S O & -0.969986 & 0.323502 & -1.145981 \\
S O & 0.065994 & -0.009491 & 1.568008 \\
C O & -2.209991 & -1.623489 & 2.136002 \\
N 0 & -2.469986 & -2.124481 & 3.164000 \\
C O & 0.661987 & -1.931488 & -2.057983 \\
N O & 0.805984 & -2.295486 & -3.163986 \\
C O & 1.515000 & -2.371475 & 0.405000 \\
O O & 2.178986 & -3.195480 & 0.859009 \\
C O & -3.386993 & -1.184479 & -0.101990 \\
O O & -4.461000 & -1.386490 & -0.463974 \\
S O & 2.336990 & 0.088500 & -0.941986 \\
C O & 3.642990 & -1.069489 & -1.406982 \\
C O & 0.271988 & 1.792496 & 1.311005 \\
C O & -0.775986 & 2.478500 & 0.473007 \\
C O & -0.740997 & 2.113495 & -0.987976 \\
C O & -1.035995 & -2.427475 & -0.016998 \\
O O & -1.162994 & -3.575485 & -0.147003 \\
H O & -2.677994 & 0.279495 & 1.149002 \\
H O & -1.352997 & 0.205505 & -2.460983 \\
H O & 2.013992 & 0.399506 & -2.239975 \\
H O & 4.461000 & -0.513489 & -1.890976 \\
H O & 3.219986 & -1.835480 & -2.075974 \\
H O & 4.013992 & -1.530487 & -0.477982 \\
H O & 1.288986 & 1.951508 & 0.901000 \\
H O & 0.261993 & 2.210495 & 2.332000 \\
H O & -0.638000 & 3.575500 & 0.552002 \\
H O & -1.778992 & 2.241500 & 0.880997 \\
H O & 0.221985 & 2.383500 & -1.459976 \\
H O & -1.564987 & 2.592500 & -1.543976 \\
& & & \\
\hline
\end{tabular}

